\title{
Early Days of Scrum in an Enterprise
}

\author{
B. Reddaiah ${ }^{\# 1}$, K. Srinivasa Rao ${ }^{* 2}$

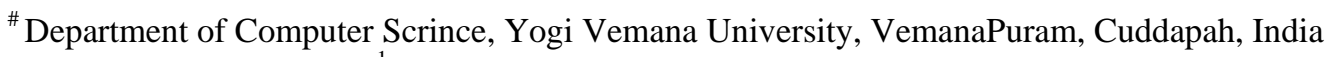 \\ ${ }^{1}$ b.reddaiah@yogivemanauniversity.ac.in \\ * Department of Computer Scrince, Yogi Vemana University, VemanaPuram, Cuddapah, India \\ ${ }^{2}$ kanususrinivas@yahoo.co.in
}

\begin{abstract}
Software developing enterprises tend to go for new process models that help them in developing software products. This is because the change in the needs of customers and the developing enterprises are not constant. To meet this old and traditional process models are not applicable. When new process models are chosen they need to be adopted first into the enterprise. After the process model is adopted it is very important to control the process model in the enterprise. It is essential to know how the process model is integrated to the environment of the software developing enterprise. The behaviour of the process model in its early period after adoption is discussed in this work.
\end{abstract}

Keyword - Agile, Scrum, Enterprise, Product development, Early Months.

\section{INTRODUCTION}

In software engineering software developing methodology is a framework that helps the developing enterprises to organize, plan, and regulate the course of developing a software product. Around 1960s for the first time these frameworks for software development appeared. In 2004 Geoffrey Elliott [7] described that these systems development life cycles for developing the products referred as SDLC. These are the eldest formal organized frameworks for developing information schemes. The key function this software development life cycle in the 1960s is to develop business oriented products in larger way.

Enterprises to develop products or to manufacture products require process models to follow in doing the work because they help in smooth running of overall activities. Process models play an important role in the life cycle of a product. Without a process model it is difficult to get a successful product as it is considered as the path for carrying the work to be done. In software engineering to develop products process models are being used from the early days. Water fall model is considered as the base model for all new models in software industry. If a new process model is to be developed it has to follow the basic concepts of waterfall model. Fig. 1 shows the phases of waterfall model which is sequential in approach. Every phase in waterfall model is processed one after the other in product development.

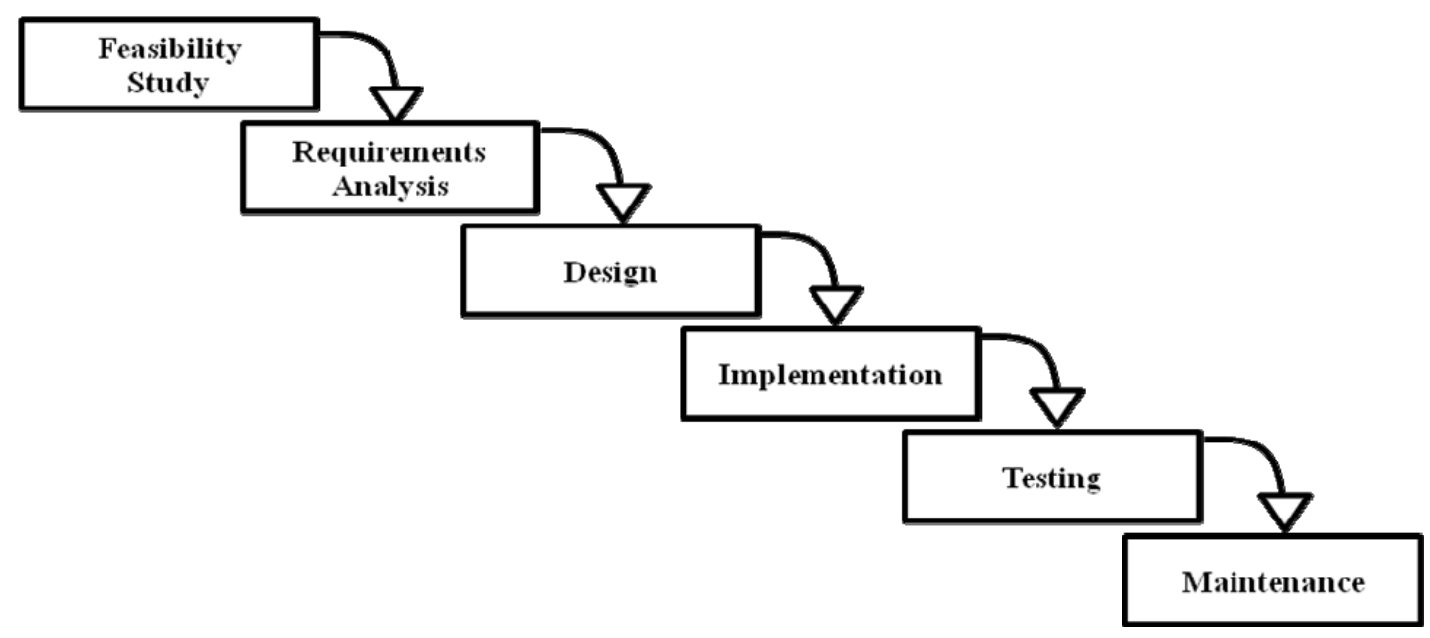

Fig. 1. Waterfall Approach [1]

Process models that exist in software engineering have it strengths and weaknesses and they are opted based on the environment of new product to be developed. They are measured based on the requirements of both the customer and developing organizations. Because of different requirements of customers and developers many process models has been developed to help in product development. In recent times a new group of methodologies has been developed that are acting as substitute to traditional and modern process models. These are agile methodologies that respond to changeability in product development. As other modern methods these agile methodologies are also iterative and incremental in nature [3]. These are suitable for developing business oriented products. Among the several agile methods the successfully and widely used methodologies are extreme programming [8] and Scrum [14]. 


\section{Motivation}

Traditional product development is replaced by Agile as a substitute that supports teams when there is changeableness in future. Compatibility is the main factor of that affects the acceptance of agile methodologies $[9,11,13]$. In the group of Agility best and common process model used in industry particularly for software development is scrum because of its ease and flexibility. Ken Schwaber first described scrum in 1996 [15].When scrum is used within a company can provide significant benefits [10] and its practices are not partial to local projects [12]. Scrum is a modest framework with operative team association that helps management and teams to satisfy commercial needs by cutting over difficulties and emphasis on satisfying customer requirements and it is a lightweight process model that is used to develop small projects. As a support to management its framework helps in dealing with violent targets, composite necessities and a grade of distinctiveness.

Scrum develops projects based on scientific procedures and it does not depend on unlearned guesses in planning and scheduling product development. Extreme programming is a set of processes that is used to develop bigger products than Scrum. Scrum framework [2] is designed in such a way that it concentrates on iterative development through which the increments of the product can yielded at the end of each and every iteration. Scrum mainly focuses on handling backlogs at various levels through different kinds of meeting [2]. The major advantage of scrum is daily meetings. Here meetings are intended to discuss about the activities that are carried. The team members participate in scrum meetings for product developments are cross functional. Each member does not have a fixed role in team. The work is carried in iterative manner.

Iteration in scrum is called sprint which is considered as the main part of software product development methodology and is shown in the Fig.2. The scrum process overview along with sprint is also shown in the Fig. 2. Actual work is carried in sprint that is considered as most important element in scrum which will last for a week to month [5]. Even though sprint has specified time period, work can be blocked at any point of time known as time boxed [6]. After completion of each sprint stakeholders and cross functional team members get together collectively. They re-evaluate the improvements in sprint called scrum cake [4]. With this meeting next steps to be taken are easily traced. If determined work is not completed time cannot be changed for sprint cycle. These sprints are recursive in character and work is carried in repeated iterations. This lets the team to alter work to be done based on finished work, but not on speculation or forecast. As the process model is very simple and gives good results for medium, small projects and involves customer which is major advantage the enterprises are moving towards scrum. For this a new model that every enterprise wants to use which they are not familiar about has to be adopted in to the enterprise [1].

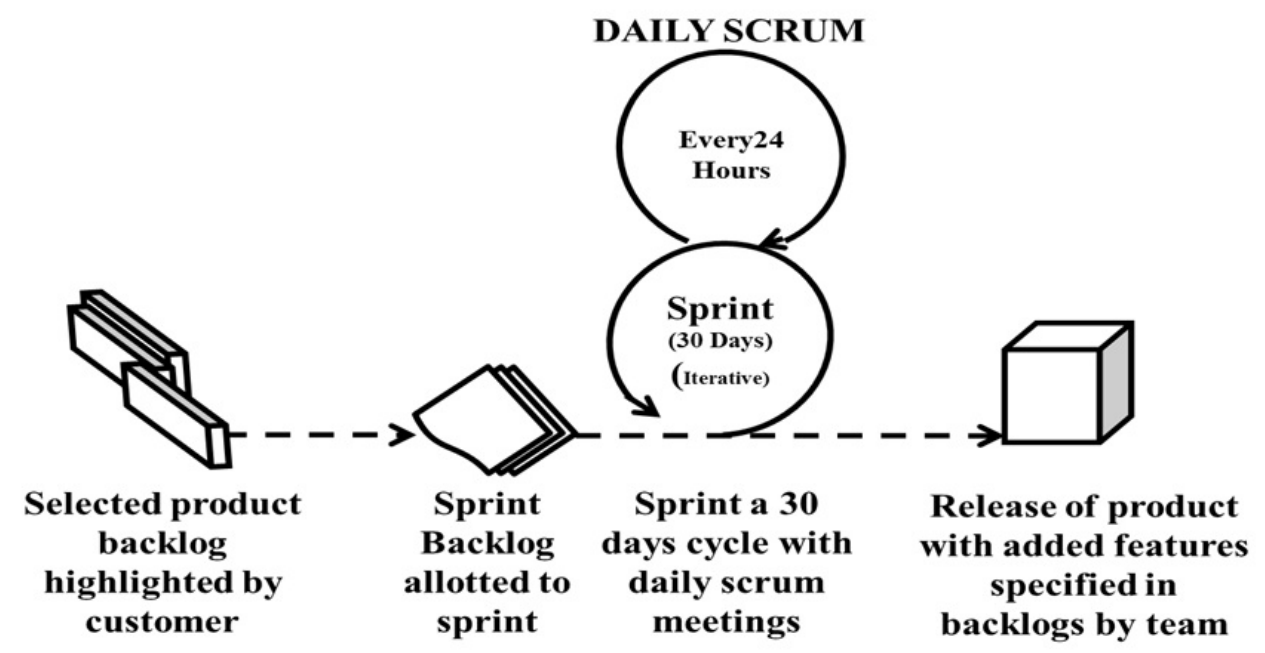

Fig. 2. Scrum Process Overview [2]

The process of adopting a new process model in to the enterprise may look easy on paper, but it is very difficult when it is handled [1]. An overall change is needed in the enterprise that is not very easily accepted by the components of enterprise. Adoption is a process with some changes in the enterprise culture and it will be new to all the members. Now every new model adopted gives rise to some real problem in using it for product development. There is no doubt that in early days of its use many difficulties have to be faced because of lack of experience in handling it both by management and by skilled people. In this work a brief discussion on Scrum is given and then detail discussion is on how the Scrum process model behaves in its early months in the software developing enterprise. And also discussion is made on eliminating the obstacles that come across. This helps in adjusting with new model very easily and quickly, so that complexity that arises in course of using new model in initial days will be handled. 


\section{III.EARLY MONTHS OF SCRUM}

Once enterprise goes for a change and think of using new process model, first it has to be adopted into the enterprise. The adoption process [1] includes preparing the entire enterprise to use new process model. The Scrum adoption process is initiated by Scrum kick-off meeting. This meeting is the first meeting conducted in enterprise. Project team along with client of the project are going to participate in this meeting and project planning activities are discussed. If there are new ream members proper preparation is to be given for upholding quality of work and standards of organization. This preparation mainly includes training people of enterprise to adjust to new culture. Then enterprise transition team (ETC) is responsible for its success. In this work first few months of scrum after adoption is discussed, because this period is very important. The adoption process might not be wonderful but flexibility will be there to go for corrections. The first month will be more exciting and confusing. Some time it spoils the morality of the people of enterprise. Enterprise has to wait until everything goes according to planning. As it goes on perfection comes to people of enterprise and problems are automatically attended.

\section{A. First Month of Scrum}

The initial part of work in first month is to conduct first Sprint planning meeting and it is referred as scrum kickoff meeting. This meeting takes less than a day. In these meeting, members for the Enterprise transition team and Scrum rollout team are to be selected to start first sprint. Here date and time for enterprise transition Sprint planning meeting is to be defined. As per scrum guidelines in this meeting scrum rollout teams has to meet with enterprise transition product owner for choosing and binding to backlogs for first sprint. It is to prepare a plan in satisfying their assurances. Enterprise transition team and scrum rollout team take part in the sprint planning meeting. A list of transition product backlogs is to be specified in the first Sprint planning meeting. These backlogs describe order of work to be carried-out in future. This list probably should consist of the following objects.

1) Make sure that every individual in the enterprise knows the reason for adoption of scrum and in what way it will be rolled out. Use modes like company meetings, departmental meetings, handouts, and video conferences to transfer the message about the importance of scrum, its effect on enterprise, and people with each individual frequently.

2) After passing on the importance, training to all the members of enterprise on scrum is to be given. In the process the reason for adoption, planning made and expected outcome is to be passed. Information should be passed to members that it is not a different and fresh methodology from the existing methodologies.

3) Convey that it is a workout procedure to uplifts the enterprise. This covey process should be two way one. Let the people of enterprise raise their doubts about scrum and its influence on the people. Their doubts should be fixed in a right manner.

4) Then create a sample problem that can be handled by using scrum. Divide the problem in to least, average and best segments. For this to happen build Transition product backlogs such that the segments can be achieved by using it.

5) With this experience enterprise can go and find actual problem that can be solved by using scrum.

6) To solve this, isolate product owner, scrum master and team that can build the product.

7) As it is new methodology that is going to be applied on product development for the first time, metrics for scrum and tools are to be collected to achieve success in development.

8) Now it is time for starting the development and product development starts with finding enterprise product backlog.

9) To carry-out all the activities with different teams and members scrum master is to be identified who acts as a coordinator between them.

10) As it is new and different to all the members encouragement plans are to be made for better team work.

11) To carry-out the work smoothly outline scum project reporting requirements which are need for product development.

12) The final thing to be set is a scrum centre that helps the team members to meet with each other as a part of meetings to discuss.

Scrum implementation process shown in Fig. 3 is used sprint followed by another sprint in adopting scrum all over the enterprise. Number of transition product backlogs will grow as scrum is widely spread throughout the enterprise. These backlogs are very well identified as impediments through which modifications are acknowledged. In course of handling these impediments enterprise, management and leaders of the enterprise transition team decides the adoption of scrum either rapidly or slowly and either smoothly or embarrassingly. With this it is obvious that adoption of scrum in enterprise is a process to change the people's culture and environment. The adoption process of scrum in enterprise has started and the first rollout sprints throughout enterprise are in progress. 


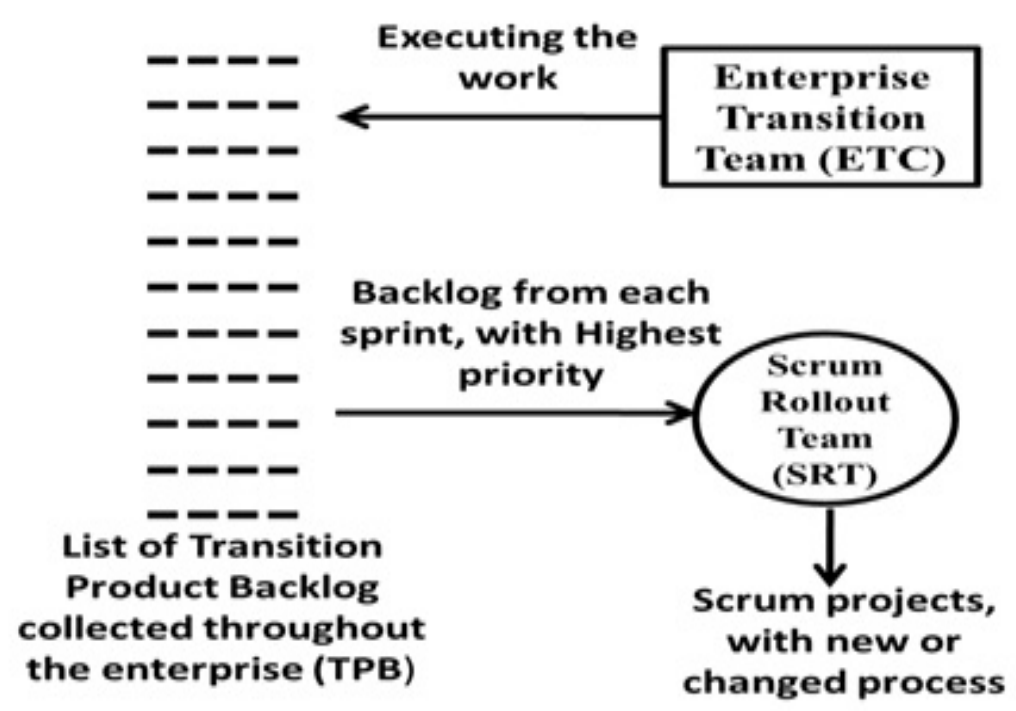

Fig. 3. Scrum adoption process with scrum implementation project-ETC[2]

\section{B. Second Month of Scrum}

The initial part of work after adoption is seen in first month and when second month comes the enterprise might have started developing several scrum projects. But starting the projects is not good without planning, proper staff and without having product backlogs. The enterprise may think in another way. They want to start the development with first sprint for different projects. When it comes to first incremental stage they find the reasons given in not starting the projects as impediments. These impediments are to be collected and they should be placed as enterprise transition product backlogs for fixing. Simultaneously along with finding and handling these impediments the scrum development teams as a part of development has to develop actual products. This software product development has to be continued without waiting for faultlessness. If work is carried in this way results cannot be expected but scrum has the strength to direct the teams to reach the goal properly.

In due course if any problem occurs that may show the effect on productivity, it is the responsibility of scrum master to handle these hurdles that come across. When it comes to reality every hurdle cannot be handled by scrum master because of lack of expertise in those issues, familiarity about the issues and possibility to fix them. In these situations it is the duty of scrum master to place such kind of issues in front of enterprise transition team's daily scrum. Once the issues are place in front of enterprise transition teams they will be fixed rapidly or they will be placed in transition product backlog for arranging them with priority and for future determination as shown in Fig. 4

Now in the enterprise as we are using scrum for first time in software product development there may be some struggles that will be faced by enterprise between old practices that are before scrum and the manner in which scrum works. The major advantage of scrum process over other is that it is extremely improved process for developing software products. With its extremeness everything is observable in the way of development. The major advantage of scum process model is that it discloses each and every deviation in the enterprise. These deviations may be the impediments that are prior to implementation of scrum or after the implementation of scrum. The problems prior to scrum implementation may be the unresolved ones or accepted ones which are not considered as major obstacles for product. After implementation of scrum with change in environment now they will be exposed that may cause problems. So they are to be handled carefully and removed. For this reason they need to be placed in transition product backlogs. 


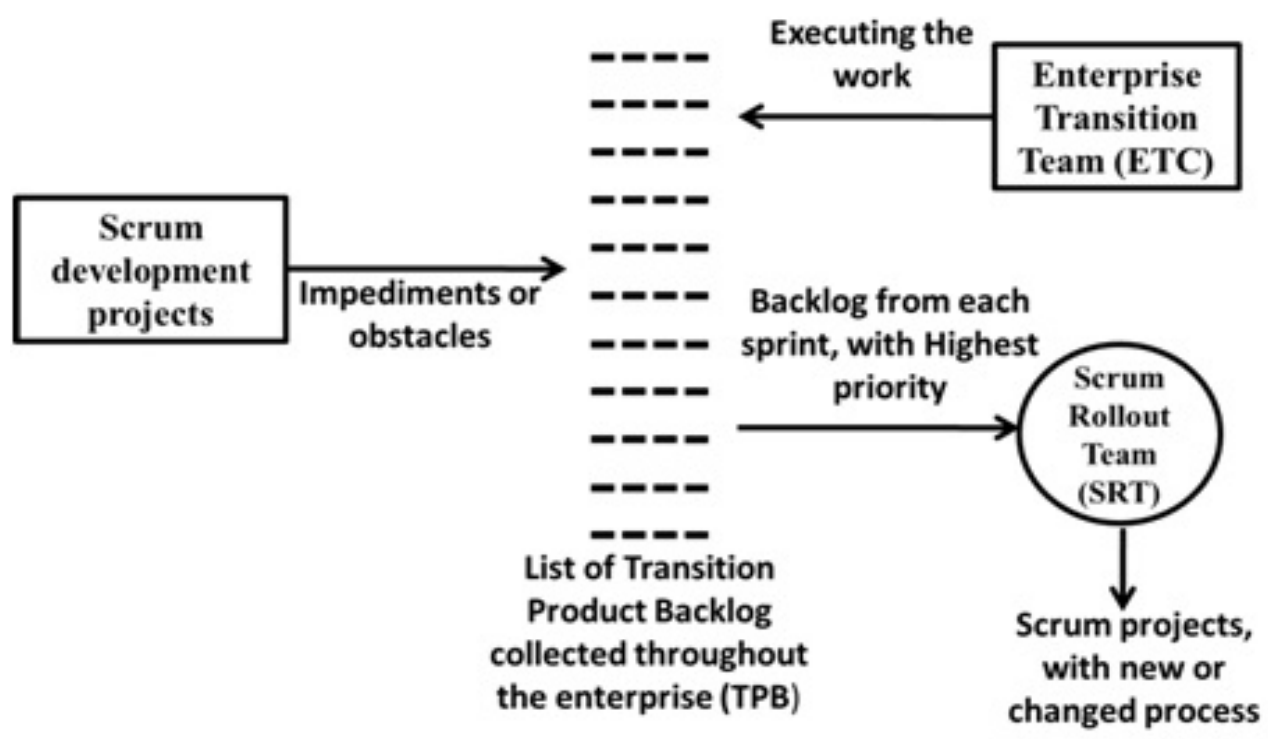

Fig. 4. Scrum Rollout [2]

As new impediments are being found every time in different cases the list of transition product backlogs will be changed repeatedly. Because of this change enterprise has to face unpredicted tasks frequently. As a part of this it is the duty of enterprise transition team to concentrates on transition product backlogs. They will be repeatedly revised and priorities will be changed to mirror the changes. This overall practice from the revision you make changing in priorities comes under adoption.

Key reasons for transition backlog impediments: The base and widely used model process model that is generally practiced in most of the enterprises is waterfall model. Development under waterfall model starts with collecting requirements for the project. The collected requirements are gradually disintegrated into different forms of phases like architecture, design, code, testing and documentation. This process is carried by professionally skilled people of that functionality. With this project development starts using waterfall model. Other than this customer also might have been accustomed to this approach, When scrum is implemented the dissimilarities between scrum attitude, practices, and behaviour is different from waterfall model. This may create big difference about the people thinking and the way of doing work. These differences that becomes as obstacles are referred as impediments and they cannot be controlled from arising. Changes in market requirement, floating of people in enterprise, stress factor are the possible reasons for impediments. When these impediments become more in number important ones should be cleared first and later comes the other impediments. This is possible by giving priority to impediments which will make work easy to process.

Role of scrum in handling impediments: Irrespective of the complexity of the impediment it is the duty of the scrum rollout teams to find the solution and directions should be given in that direction. In this activity it is enterprise that should support all the teams mainly rollout teams, so transition product backlogs should be visible to all in the enterprise. This will reduce the pressure on teams and with this regrouping is possible between teams to increase productive.

When scrum is used to develop projects enterprise transition teams takes charge right from adoption. During this process if impediments come as hurdles it is the duty of rollout teams to process them. If rollout teams fail to control the impediments in some situations it is due to lack of right composition in the team. When this happens it is time to review the team's structure and rollout teams commit to make changes. If the change that is intended in rollout team is very big it is better to ask the teams to decompose the modification into actionable fragments and transfer the transition product backlog element to other sprint. Sometimes the changes that are to be made may be more in number. In these cases everything cannot be done at a time. For this a constant improvement in team organization is needed by looking back frequently and by saving changes. This takes some time to handle impediments and patience is required.

\section{Third Month and Beyond with Scrum}

With the completion of two months after bringing scrum into the enterprise, it is easy now to differentiate your obstacles and the enterprise obstacles with which problems are being faced. With the experience of two months divide and see the obstacles. Put the obstacles that scum is creating after its adoption on one side and on the side keep the obstacles that are there before the adoption of scrum and still continuing which are highlighted by scrum. In most of the cases second one carries more obstacles that the enterprise is facing. If the second side goes on increasing which enterprise might have taught before the adoption of a new process model enterprise's 
capability to build and organize modest products may be reduced. With such problem arising generally people in enterprise may suggest to change scrum because it wishes to have certain modification to adjust with the new enterprise. It is very good to stick on with scrum because the guidelines, characters and time-boxes of scrum are very less and modest. This expose of problems when scrum is used because of its practices and structure that discloses unsolved impediments. General trend that everyone feels is one should not expose the problems of their own and they go for changing scrum. But if scrum is changed the impediments remains unsolved and suppressed that shows their impact on productivity in future.

Another important that enterprise should focus is that when scrum is being implemented enterprise transition team is going to make change in the culture of developing products. So the enterprise has to find right ways to develop products between old way of developing and new way of developing. There should be a clear workout to confirm that accommodations are short-term. Care should be taken not in making them long-lasting ways of doing business. In some cases enterprises think of bringing scrum tools to be well-matched with the enterprise existing carry outs. The intention of enterprise may be to say that the change can be made in smooth way. But there is every possibility by the people of enterprise to think that change is not made in a serious way. This will not help the enterprise in developing new terminology and seriousness in people towards new terminology will be lacking which hurts enterprise transition. Now with scrum enterprise can obviously observe the influence of scrum on a project's growth at the sprint review that is conducted for every 30 days. One has to understand in this stipulated time everything that is committed cannot be finished. Some problems require more than one attempt to find the solution. While using scrum it is to notice when a solution to a problem isn't good or when a problem is changing its shape, rewriting the solution is needed. So, perfect solution is not necessary. This can be achieved sprint after the sprint with carry-outs into the next sprint.

To achieve success people and the management has to come-out with good practices. People should be made to recognize the causes for adoption of scrum. When people know the benefits of change in their daily work then it will be easy to them to understand. For this to happen visit nearby enterprises and their people who had effectively succeeded in adopting and using scrum in product development. By observing enterprises that are successfully using scrum new enterprises can develop and adopt metrics to get inspired and track modification. But every care should be taken because that these metrics may show unsuspected consequences on other parts of work. For all this happen it will take some time to show its impact and the enterprise has to wait for better results.

\section{CONCLuSION}

Products that are developed are to satisfy the needs of customer. The framework that is useful to developing enterprises is a process model. There exist many process models from conventional to modern process models. The enterprise has to select a right process model that suits the enterprise, teams and the customer. Scrum process model is very extensively used in developing small and medium products. With this process model success rate and satisfaction of customer is up to maximum extent. When a new process model is developed and if the enterprise wants to bring that model in to it, the work is of two types. First the new process model has to be adopted and after adoption the process model must fit in the culture of the enterprise. Post adoption work after adopting scrum process model is discussed in this work. This behaviour of new process model while developing is categorically discussed as first month, second month and third month of scrum in enterprise. The behaviour of scrum and its influence on people in early days after adoption is made clear in this study. The role of the enterprise and the people of enterprise in handling scrum is clearly discussed. This helps the enterprises to effectively handle scrum process model after adoption. The role of enterprise transition team and rollout teams is clearly in handling the product development is discussed. The important aspect is handling the impediments that come when process model is adopted and while developing the project is also discussed. The precautions that are to be taken from getting new troubles when new way of development undergoes is mentioned. When good practices are made and habituated they set the enterprise in the right direction of excellence.

\section{ACKNOWLEDGMENT}

We sincerely thank to Dr. Lakshmi Sridhar Movva for his cooperation in doing this research. He is certified scrum master and has been practicing scrum in the projects he executed. We specially thank Rajasekhar Kilaparthi a Consultant in Information Technology, Scrum Master and a Certified PRPC Senior System Architect. He has rich experience in IT industry. He is a Scrum Master and has been practicing Scrum in the projects. 


\section{REFERENCES}

[1] B. Reddaiah, R. Pradeep Kumar Reddy, C. Nagaraju, V. HarshaSree, “A novel approach to adopt scrum by an enterprise,” Advances in Intelligent Systems and Computing,: Springer India, 2016, vol.,pp.645-654.

[2] B. Reddaiah, Satya Prasad Ravi, Lakshmi Sridhar Movva, "Risk Management Board for Effective Risk Management in Scrum," International Journal of Computer Applications(IJCA), vol.65, no.12, pp.16-23, 2013.

[3] B. Reddaiah, M. Padmaja, P. Vishnupriya, K. Surekha, "Handling transition product backlog with scrum off scrum," International Journal of Advanced Information Science and Technology(IJAIST), vol.45, no.45,pp123-126, 2016.

[4] Dr. Satya Prasad Ravi, B. Reddaiah, Lakshmi Sridhar Movva, Rajasekhar Kilaparthi, "A critical review and empirical study on success of risk management activity with respect to scrum,” IRACST-Engineering science and Technology: An International Journal(ESTIJ), vol.2, no.3, pp.467-473, 2012.

[5] Schwaber K (2004), Agile project management with scrum, Microsoft press, Redmond. ISBN 978-0-7356-1993-7.

[6] Sprint Planning (January-February 2009). Sprint Planning Rules. Retrived March 30, 2009.

[7] Gepffrey Elliott (2004) Global Business Information Technology: An integrated systems approach. Pearson Education.p.87.

[8] K. Beck, Extreme Programming Explained: Embrace Change, Addison-wesley Longman Publishing Co., Inc., USA, 1999.

[9] A. Cockbum, J. Highsmith, Agile software development: the people factor, IEEE computer 34(11), 2000, pp.131-133.

[10] C. Mann, F. Mauer, A case study on the impact of scrum on overtime and customer satisfaction: Agile development conference, 2005, pp. 70-79.

[11] J. McManus Team agility, Computer Bulletin 45(5), 2003, pp.70-79.

[12] A. Danait, Agile offshore techniques-A case study: Agile Conference, 2005, pp.214-217.

[13] B. Schatz, I. Abdelshafi, Primavera gets agile: a successful transition to agile development, IEEE software 22(3), 2005, pp.36-42.

[14] K. Schwaber, SCRUM development process: Proceedings of the conference on object-oriented programming systems, Languages and applications workshop on business object design and implementation, pp117-134, 1995.

[15] Advanced Development Methods (1996) Controlled chaos: Living on the edge, http://www.controlchaos.com/old-site/ap.html.

\section{AUTHOR PROFILE}

B. Reddaiah is working as Assistant Professor in Department of Computer Science, Yogivemana University since 2009. He completed Ph.D from Acharya Nagarjuna University in 2015 under the esteemed guidance of Dr R.Satya Prasad in Computer Science and Engineering. He Published 18 International papers and attended 6 National and International conferences at various places in India. The areas of research are Software Engineering, Cryptography and Network Security.

K. Srinivasa Rao is working as Assistant Professor in Department of Computer Science, Yogivemana University since 2009. He is doing his Ph.D from Acharya Nagarjuna University. He Published 10 International papers and attended 4 National and International conferences at various places in India. The areas of research are Digital Image Processing, Cryptography and Network Security, Software Engineering. 